( $\rho=-0.260, p=0.015)$, moreover, positive correlation with serum LPS $(\rho=0.284$, $p=0.008)$ and LBP $(\rho=0.247, p=0.021)$ and inverse correlation with ENC ( $\rho$ $=-0.321, p=0.002$ ) were found. On the other hand, IgG anti-Pg-LPS antibody levels which reflect systemic infection, inversely correlated SDAI $(\rho=-0.308, p=$ $0.004)$, PainVAS $(\rho=-0.433, p<0.0001)$, and so on.

Figure 1. Relationships between intestinal bacterial population and disease activity, Disease marker, LPS related marker and anti-Pg LPS antibody levels

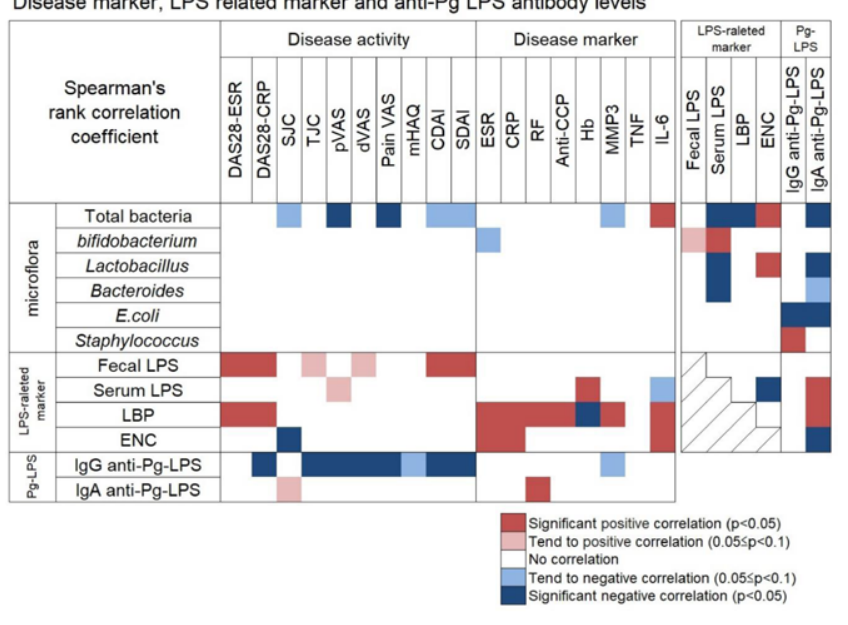

Conclusion: Serum LBP highly associated with RA activity and markers, which suggests bacterial LPS as roles in triggering and perpetuating disease activity in RA. In contrast, IgA anti-Pg-LPS, IgG anti-Pg-LPS antibody reflecting infection of $\mathrm{Pg}$, negatively associated with intestinal total bacteria $(\rho=-0.4405, p<0.0001)$, RA disease activities, respectively. These results may show a possible oral -gut relationship resulting in aggravation of disease activity in RA.

Disclosure of interest: None declared

References:

[1] Sato K, Takahashi N, Kato T et al. Aggravation of collagen induced arthritis by orally administered Porphyromonas gingivalis through modulation of the gut microbiota and gut immune system. Sci Rep 2017; 7:6955.

[2] Kuniaki Terato, Takaki Waritani, Richio Fukai et al. Contribution of bacterial pathogens to evoking serological disease markers and aggravating disease activity in rheumatoid arthritis. PloS One. 2018;13(2): e0190588.

Disclosure of Interests: None declared

DOI: 10.1136/annrheumdis-2020-eular.1368

\section{THU0067 JAK SELECTIVITY AND THE IMPACT ON CYTOKINE SIGNALING INHIBITION AT CLINICAL RHEUMATOID ARTHRITIS DOSES}

P. Gonzalez-Traves ${ }^{1}$, B. Murray ${ }^{1}$, F. Campigotto ${ }^{1}$, A. Meng ${ }^{1}$, J. A. DI Paolo ${ }^{1}$. ${ }^{1}$ Gilead Sciences, Inc., Foster City, United States of America

Background: Janus kinase 1 (JAK1) inhibitors are efficacious in rheumatoid arthritis (RA). Despite having similar efficacy, in vitro studies have shown differences in JAK selectivity profiles for the small-molecule JAK inhibitors (JAKi) baricitinib (BARI), tofacitinib (TOFA), and upadacitinib (UPA). ${ }^{1}$ For example, BARI and UPA are JAK1/JAK2 selective, while TOFA is JAK1/JAK3 selective, but each JAKi has some activity against other JAKs. As JAKs form signaling pairs, differences in selectivity could lead to distinct pharmacologic profiles that may impact clinical efficacy and safety.

Objectives: As a first step to understand the basis of potential differences at therapeutic doses, we compared the selectivity and potency of filgotinib (FIL) and its major metabolite (MET) to those of BARI, TOFA, and UPA in cytokine-stimulated peripheral blood mononuclear cells (PBMCs) and whole blood (WB).

Methods: PBMCs and WB from healthy donors were incubated in vitro with 8 doses of each JAKi, and levels of signal transducer and activator of transcription phosphorylation (pSTAT) were measured following cytokine stimulation. Half maximal inhibitory concentration $\left(\mathrm{IC}_{50}\right)$ values were calculated in phenotypically sorted leukocyte populations by flow cytometry. Therapeutic dose relevance of the in vitro analyses was assessed using calculated mean concentration-time profiles from JAKi population pharmacokinetic data in RA subjects. For each JAKi, the time above $I C_{50}$ and average daily pSTAT inhibition were calculated for each cytokine/STAT pair in B cells, CD4+ T cells, CD8+ T cells, monocytes, and/or NK cells.
Results: Cellular assays in PBMCs and WB showed dose-dependent inhibition of cytokine-induced pSTATs with all JAKi (correlation between the protein-adjusted $\mathrm{IC}_{50}$ values from PBMCs and $\mathrm{IC}_{50}$ values from $\left.\mathrm{WB}, \mathrm{r}^{2}=0.98\right)$. Among the most potently inhibited pathways were JAK1/TYK2-dependent cytokine, interferon alpha (IFNa), and the JAK1/2-dependent cytokine, interleukin (IL)-6. FIL and MET had weaker potencies against JAK2/TYK2 (G-CSF/pSTAT3), JAK1/2 (IFNy/pSTAT1), and JAK2/2 (granulocyte-macrophage colony-stimulating factor [GM-CSF])-dependent pathways compared to JAK1/TYK2 (IFNa/pSTAT5). FIL and MET showed the greatest selectivity vs the JAK2/2 pathway (GM-CSF/ pSTAT3) in monocytes.

The mean concentration-time profiles and time above $\mathrm{IC}_{50}$ over $24 \mathrm{hr}$ for each cytokine/STAT pathway showed that JAK1/2 (IL-6/pSTAT1) and JAK1/TYK2 (IFNa/pSTAT1) pathways were strongly modulated with all tested JAKi. FIL $(200 \mathrm{mg})$ showed similar activity in average target coverage and time above $\mathrm{IC}_{50}$ to the approved low doses of TOFA $(5 \mathrm{mg})$ and UPA $(15 \mathrm{mg})$; conversely, FIL had reduced mean average inhibition and time above $I_{50}$ levels against JAK1/2 (IFNy/pSTAT1), JAK1/3-dependent cytokines (IL-2, -4, and -15), JAK2/ TYK2 (G-CSF/pSTAT3), and JAK2/2 (GM-CSF/pSTAT5)-dependent pathways compared to TOFA and UPA, and in certain cases to BARI $(2 \mathrm{mg})$.

Conclusion: Different JAKi modulate distinct cytokine pathways to varying degrees, and no agent potently and continuously inhibited an individual cytokine signaling pathway throughout the dosing interval. FIL $(200 \mathrm{mg})$ showed a similar inhibition profile to TOFA, BARI, and UPA against the JAK1/TYK2- (IFNa/ pSTAT1) or JAK1/2-dependent (IL-6/pSTAT1) responses, consistent with the role of these pathways in clinical efficacy. ${ }^{2}$ However, FIL displayed a differentiated pharmacologic profile from the other JAKi, showing biologically reduced activity on the JAK1/2 (IFNY)-, JAK1/3 (IL-2, -4 and -15)-, JAK2/TYK2 (G-CSF)-, and JAK2/2 (GM-CSF)-dependent pathways, which play important roles in hematopoiesis and immune function. These data suggest that FIL $(200 \mathrm{mg})$ may have less impact on a subset of homeostatic immune functions signaling via JAK2 and JAK3 than those observed at the clinically approved doses of TOFA (5 mg and $10 \mathrm{mg})$, UPA (15 mg), and BARI (4 mg).

References:

[1] Mclnnes IB, et al. Arthritis Res Ther. 2019;21:183.

[2] Banerjee S, et al. Drugs. 2017;77:521-546.

Disclosure of Interests: Paqui Gonzalez-Traves Employee of: Gilead, Bernard Murray Employee of: Gilead, Federico Campigotto Employee of: Gilead, Amy Meng Shareholder of: Gilead Sciences, Employee of: Gilead, Julie A. Di Paolo Employee of: Gilead

DOI: 10.1136/annrheumdis-2020-eular.2074

\section{\begin{tabular}{|l|l}
\hline THU0068 COMPARISON OF INFLAMMATION DURING \\
\hline
\end{tabular} METABOLIC CHANGES IN RHEUMATOID AND OSTEOARTHRITIS MOUSE MODELS}

H. M. Sauermilch ${ }^{1}$, M. L. Hülser ${ }^{1}$, C. Schreiyäck ${ }^{1}$, Y. Luo ${ }^{2}$, A. Bozec ${ }^{2}$, G. Schett $^{2}$, U. Müller-Ladner ${ }^{1}$, E. Neumann ${ }^{1}$. 'Dept. of Rheumatology and Clinical Immunology, Campus Kerckhoff, Justus-Liebig-University Giessen, Germany, Giessen, Germany; ${ }^{2}$ Department of Internal Medicine 3, Friedrich Alexander University of Erlangen-Nuremberg and Universitatsklinikum Erlangen, Erlangen, Germany, Erlangen, Germany

Background: Arthritis is influenced by metabolic changes. Adipokines are bioactive factors produced by adipose tissue with important effects on energy homeostasis and immune responses but are also involved in the pathogenesis of rheumatoid arthritis (RA) and osteoarthritis (OA).

Objectives: To evaluate inflammation during metabolic and adipokine induced changes in experimental models of RA and OA at different time points, an obesity model (high-fat-died, HFD) was therefore combined with an OA (DMM, destabilization of the medial meniscus) and RA (collagen induced arthritis, CIA) mouse model.

Methods: Mice were fed with HFD or ND (normal diet) for 12 (OA) or 6 (RA) weeks prior to arthritis induction. DMM was performed in $\mathrm{C} 57 \mathrm{BI} / 6$ mice and CIA was induced in DBA/1Rj mice. After 4, 6 and 8 (DMM) or after 4, 5 and 7 weeks (CIA) of arthritis induction animals were sacrificed to collect histological and serological data. Clinical scoring for CIA and histological scorings for both models were performed to evaluate disease development and progression Diet-induced effects were monitored by weight, fatty liver score and crown-like structures (CLS) counts in adipose tissue. To evaluate HFD-induced adipokine levels adiponectin, leptin and visfatin serum concentrations were measured in parallel to the CRP. Local tissue adipokine expression was evaluated by immunohistochemistry.

Results: Induction of OA and RA was successful in an HFD setting, shown by histological joint destruction and the increased fatty liver score and bodyweight. Compared to healthy animals, CRP levels were significantly increased after $\mathrm{CIA}$ induction, confirming systemic inflammation. In DMM, the number of CLS 
were significantly higher in HFD $(0.2 \pm 0.16, \mathrm{n}=7)$ compared to ND $(5.2 \pm 0.98$, $n=8$ ). No difference was found in CIA-severity between HFD and ND. However, $\mathrm{CIA}$ induction increased the number of CLS in HFD $(2.77 \pm 1.07, \mathrm{n}=6)$ and interestingly in ND animals $(8.14 \pm 0.23, n=5)$ compared to healthy ND $(0.45 \pm$ $0.03, n=4)$ and healthy HFD mice $(2.57 \pm 0.53, n=4)$ without CIA. As expected, HFD led to a significant increase in systemic leptin in healthy animals in both models. Interestingly, CIA and DMM induction decreased systemic leptin levels significantly in ND and HFD, which was more prominent in CIA. The systemic effect was not reflected by local leptin distribution in the joints $(\mathrm{CIA})$ which were not altered by diet. 5 . and 7 weeks after CIA induction HFD led to a reduction in local adiponectin and visfatin expression, which were not reflected in systemic levels.

Conclusion: The data show that HFD deteriorates OA, which is similar to observations in humans. In contrast, HFD induction showed no significant difference in $\mathrm{CIA}$ severity compared to ND. Furthermore, CIA reduced local adipokine expression under HFD at later time points but not under ND. According to high numbers of CLS in ND/CIA animals and the strong reduction of leptin in CIA with HFD, $\mathrm{CIA}$ onset and severity seems to be obesity independent and more dependent on inflammation while OA appears to be directly influenced by obesity. However, HFD-induced obesity seems to alter local and systemic adipokine expression also in CIA. Interestingly, local adipokine distribution in affected joints was independent from systemic adipokine levels.

Disclosure of Interests: Hani Manfred Sauermilch: None declared, Marie-Lisa Hülser: None declared, Carina Schreiyäck: None declared, Yubin Luo: None declared, Aline Bozec: None declared, Georg Schett Speakers bureau: AbbVie, BMS, Celgene, Janssen, Eli Lilly, Novartis, Roche and UCB, Ulf Müller-Ladner Speakers bureau: Biogen, Elena Neumann: None declared

DOI: 10.1136/annrheumdis-2020-eular.2898

\section{THU0069 MIMICKING ARTHRITIS IN VITRO TO TEST DIFFERENT TREATMENT APPROACHES}

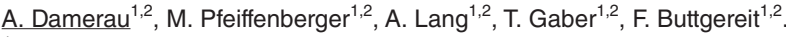
${ }^{1}$ Charité - Universitätsmedizin Berlin, Corporate Member of Freie Universität Berlin, Humboldt-Universität zu Berlin, and Berlin Institute of Health, Department of Rheumatology and Clinical Immunology, Berlin, Germany; ${ }^{2}$ German Rheumatism Research Centre (DRFZ) Berlin, a Leibniz Institute, Berlin, Germany, Berlin, Germany

Background: Our ultimate goal is to study potential drug candidates in an experimental setting of arthritis. Therefore, we aim to develop a valid human in vitro 3D joint model mimicking features of joint inflammation by applying inflammatory conditions namely immune cells and pro-inflammatory cytokines. Our in vitro 3D joint model consists of different components including an osteogenic and chondrogenic part, the joint space filled with synovial fluid, and the synovial membrane. Developed as an alternative experimental setup to animal experiments, our 3D joint model will enable us to study efficiently the effects of potential drug candidates in a human-based in vitro model.

Objectives: Here, we aimed to demonstrate the suitability of our human-based in vitro 3D osteochondral model by analyzing the influence of the main cytokines involved in the pathogenesis of RA as well as the impact of a specific therapeutic intervention.

Methods: Based on human bone marrow-derived mesenchymal stromal cells (hMSCs), we developed 3D bone and cartilage tissue components that were characterized in detail (e.g. cell vitality, morphology, structural integrity) using histological, biochemical and molecular biological methods as well as $\mu C T$ and scanning electron microscope (SEM). In brief, to establish the osteogenic component, we populated $\beta$-tricalcium phosphate (TCP) - mimicking the mineral bony part - with hMSCs, while the scaffold-free cartilage component was generated by cellular self-assembly and intermittent mechanical stimulation (fzmb $\mathrm{GmbH}$ ). Subsequently, we co-cultivated both tissue components for three weeks to generate an interconnected 3D osteochondral model. To test the suitability, we applied a cocktail of TNFa, IL-6 and MIF using concentrations reported from RA synovial fluid alone or in combination with specific therapeutic drugs and analyzed their impact by qPCR.

Results: We verified the osteogenic phenotype of our 3D bone tissue component by demonstrating an increase in mineralized bone volume and the induction of bone-related gene expression (RUNX2, SPP1 and COL1A1) as compared to the corresponding control. Secondly, we verified the chondrogenic phenotype of our cartilage tissue component by $\mathrm{HE}$ and Alcian Blue staining as well as by the reduced expression of COL1A1 and an abundant expression of COL2A1. Interestingly, co-cultivation of both components for up to 3 weeks demonstrated colonization, connectivity and initial calcification implying a transitional bridging area. Cytokine stimulation with a cocktail of TNF, IL- 6 and MIF leads to an upregulation of the metabolic marker LDHA and the angiogenic marker VEGF in both bone and cartilage. The inflammation markers IL8 and TNF are also upregulated in both components, while IL6 is downregulated in bone compared to the unstimulated control. In addition, a cytokine-induced upregulation of matrix-metalloproteases was observed especially in the cartilage component. All these cytokine-related effects could be antagonized with a cocktail of therapeutics (milatuzumab, adalimumab and tocilizumab).

Conclusion: The results of our study showed cytokine related effects of both tissue components, which can be therapeutically antagonized. By combining the components in a 96 well format, we aim to provide a mid-throughput system for preclinical drug testing.

Acknowledgments: This project is funded by the Federal Ministery of Education and Research (BMBF)

Disclosure of Interests: Alexandra Damerau: None declared, Moritz Pfeiffenberger: None declared, Annemarie Lang: None declared, Timo Gaber: None declared, Frank Buttgereit Grant/research support from: Amgen, BMS, Celgene Generic Assays, GSK, Hexal, Horizon, Lilly, medac, Mundipharma, Novartis, Pfizer, Roche, and Sanofi.

DOI: 10.1136/annrheumdis-2020-eular.3051

\section{THU0070 \\ DEFINING SYNOVIAL SIGNATURES IN THE RAT CIA MODEL: WHAT CAN WE LEARN ABOUT RA PROGRESSION?}

D. Ruelas ${ }^{1}$, R. LI ${ }^{1}$, C. Franci ${ }^{1}$, V. Lira ${ }^{1}$, D. Lopez ${ }^{1}$, L. LI ${ }^{1}$, G. Min-Oo ${ }^{1}$, J. A. DI Paolo ${ }^{1} .{ }^{1}$ Foster City, Foster City, United States of America

Background: Patients showing inadequate or no response to current therapies represent a key unmet need in rheumatoid arthritis (RA). To address this, novel or combination therapies are of high clinical interest. Identification of novel therapeutic targets requires a greater understanding of the pathogenic molecular drivers in the RA synovium. However, our current knowledge of human molecular patterns that emerge as a result of disease progression is complicated by patient-to-patient heterogeneity and access to synovial tissue.

Objectives: Here we use the current knowledge of human synovial heterogeneity to conduct a longitudinal study of global molecular responses in the rat collagen-induced arthritis $(\mathrm{CIA})$ model to better understand synovial biology, improve the preclinical modeling of human disease, and discover novel targets for RA.

Methods: A rat CIA model was performed as previously described. ${ }^{1}$ RNA-Seq was performed on 56 knee synovial tissues collected at multiple time points throughout the course of disease. Differential gene expression was determined at each individual time point and longitudinally with disease progression. Published human synovial datasets were used to categorize these genes into myeloid, lymphoid, fibroid, and low inflammatory signatures. ${ }^{2}$ Differentially expressed genes (DEGs) at each time point were compared to human synovial datasets of RA patients before and after treatment. In addition, we compared disease-driven genes in CIA to genes in RA patients that are unchanged following therapy to identify possible combination therapies.

Results: Disease pathology in the rat CIA natural history study progressed as expected: significant decreases were seen in body weight, as well as increases in ankle diameter, paw weight, and histopathology scores of joints in collagen-injected vs noninjected rats. There were 1900 DEGs identified between diseased and naïve rats over the course of disease, representing disease-induced gene signatures (Fig. 1). Comparing these DEGs to reported human RA synovial signatures, both the lymphoid and myeloid signatures were found to be highly upregulated. Interestingly, there were no significant DEGs representing the human fibroid and low inflammatory synovial signatures identified in the CIA rat model. This suggests that the rat CIA model most closely models RA patients with an immune synovial phenotype. In addition, we examined the overlap between disease-driven genes in CIA and genes in RA patients that are unchanged following therapy to identify signaling pathways that may be of utility in combination therapy. Of genes that were upregulated in $\mathrm{CIA}, 94 \%$ of genes that mapped to extracellular matrix-receptor pathways remained unchanged in the synovial tissue of RA patients following tocilizumab treatment.

Conclusion: Previous studies have shown that nearly $30 \%$ of treatment-naïve early RA patients exhibit a strong fibroid phenotype that correlates with less severe disease and a relatively poor response to disease-modifying anti-rheumatic drugs. ${ }^{3}$ These data indicate that the synovial biology associated with such patients (fibroid or pauci-immune) is not well captured in CIA, the most common preclinical RA model. To assess potential new therapies targeting these patients it will be necessary to develop alternative animal models with more intact fibroid signatures. In addition to these findings, we also characterized the global molecular changes that occur with disease progression in the $\mathrm{CIA}$ rat and made a comparison to RA patients on treatment, providing an overall understanding of 